\title{
Measuring Health-Related Productivity Loss
}

\author{
Rebecca J. Mitchell, MPH and Paul Bates, MB, BS
}

\begin{abstract}
The objective of this study was to determine the relationship between health status and productivity loss and to provide estimates of the business implications of lost work performance. Health risk appraisal responses from over 1 million participants were analyzed to determine productivity loss associated with several common health conditions and health risks. Propensity scores and a matching technique were used to create analysis groups that differed only by presence of a particular health condition or risk. Results were monetized and multiplied by the average number of employees with conditions or risks to illustrate the potential impact of productivity loss to employers. Costs of productivity loss were compared to medical costs for the same conditions and health risks. Practical benchmarks of lost work performance may help employers assess the financial impact of suboptimal health in their own companies. Estimates of lost work time can help employers realize the value of maintaining a healthy population. (Population Health Manage-
\end{abstract} ment 2011;14:93-98)

\section{Introduction}

D ECREASED ON-THE-JOB PRODUCTIVITY AND EMPLOYEE ABSENCE because of health result in significant costs to employers above and beyond medical spending. ${ }^{1,2}$ Health-related work losses are estimated to cost US employers more than $\$ 260$ billion each year, and may cost some companies more than direct medical expenditures. ${ }^{3,4}$

Even though research shows that companies with successful health and productivity programs have superior business outcomes and financial returns, methods to measure productivity differ and a validated method to monetize costs associated with lost work time, especially presenteeism, is still lacking. ${ }^{2,3,5}$ As a result, available estimates of productivity loss because of health conditions vary widely and employers may be uncertain about where they should focus employee health management strategies. $^{2,6}$

The objectives of this study were to estimate productivity losses for workers with health conditions realistically, and to determine the potential costs of health-related productivity loss over a 12-month period. By examining a large sample of health risk appraisal (HRA) data and matching those workers with health conditions to similar workers without health conditions, we quantified the productivity impact associated with multiple health conditions and risk factors.

\section{Methods \\ Evaluation design and sample}

To estimate productivity loss associated with health conditions or lifestyle health risks, data were obtained from more than 1.3 million employed OptumHealth HRA participants who completed at least 1 survey between January, 2007 and December, 2009. Average annual rates of absenteeism (days of work missed because of illness) and presenteeism (days at work but limited in performing job tasks because of health) for a group of individuals with health conditions or health risks were compared with a matched group of individuals without the same health conditions or risks. The propensity score analytic approach was used to avoid selection bias because randomizing the administration of HRAs was not feasible.

Data records were considered complete if they contained valid responses and identifying information, and were excluded for participants younger than age 18 or older than age 70 years at the time of the survey. After exclusions, 1 million individuals completed a survey at least once (T1 or baseline) during the study period, and nearly 260,000 also completed a follow-up survey (T2). Participants were an average of 42 years old; $77 \%$ were white and $58 \%$ were female.

\section{Measures}

The HRA instrument was developed, validated, and administered by the University of Michigan, Health Management

OptumHealth Culture of Health Institute, Golden Valley, Minnesota. 
Research Center, Ann Arbor, MI. The survey included questions about work loss related to health, the presence or absence of several chronic diseases, as well as questions concerning lifestyle or behavioral factors (eg, cigarette smoking, use of alcohol, seat belt use) and health or biological factors (eg, blood pressure, total cholesterol, height, weight) associated with health status. All measurements, including blood pressure, height, weight, and total cholesterol, were self-reported. Self-reported recall of health care utilization and health-related absence from work has been found to be reliable and valid, particularly when recall periods are short. ${ }^{7}$

Thirteen health conditions were examined for this study: allergies, arthritis, asthma, back pain, bronchitis, cancer, depression, diabetes, heartburn, heart disease, migraine, osteoporosis, and pain. Participants were asked to indicate whether they had a condition by choosing the options "never," "in the past," or "have currently." If participants reported having a condition, they were also asked to indicate whether they were currently under medical care and/or taking medications for the condition. For each health condition, individuals were assigned a value of " 1 " if they reported currently having the condition and " 0 " if they reported never having the condition or having it in the past.

Lifestyle health risks analyzed for this study included overweight/obesity, cigarette use, high blood pressure, and high cholesterol. (Other health risks measured but not included in productivity loss analyses included seat belt use, physical activity, perception of health, stress, use of relaxation drugs, life satisfaction, job satisfaction, number of medical risks, and alcohol use.) Table 1 shows these risk factors along with the established criteria used to determine whether a participant was at high risk. A binary health risk variable was created for each risk factor by assigning individuals a value of " 1 " if high risk criteria were met and " 0 " if the risk was absent. In addition, a variable for total number of risks was created by summing risk factors, and the number of risks was categorized into low (defined as 0 to 2 risks), medium ( 3 to 4 risks), and high risk (5 or more risks).

Productivity loss related to absenteeism was measured using the question: "In the past year, how many days of work have you missed due to personal illness?" Response choices were "0 days," "1-2 days," "3-5 days," "6-10 days," "11-15 days," or "16+ days." A separate question measured productivity loss related to caring for ill family members and was not analyzed for this study. A continuous variable was created by recoding response options to indicate total work days lost per year because of absenteeism (eg, "1-2 days" was recoded to 1.5 days, "3-5 days" was recoded to 4 days).

The most common approach to measuring presenteeism is to ask employees how much their health hinders their performance while at work. ${ }^{3}$ For this study, presenteeism was measured with the 8-item short form of the Work Limitations Questionnaire (WLQ), a subset of the original 25-item version, and percent productivity loss was calculated using methods outlined by Lerner and colleagues. ${ }^{8}$ In a 2004 review of 6 self-reported productivity loss instruments, the WLQ was one of 2 instruments reported to offer a significant advantage over others as a result of extensive testing and the ability to measure general health impact and impact of specific conditions. ${ }^{9}$ Research has shown that the WLQ has relatively strong validity and reliability, has been used in a variety of workplace settings with a variety of health risks and conditions, and may be most useful in general employee populations. $^{10}$

Productivity loss related to presenteeism was measured with the question: "In the past 2 weeks, how much of the time did your physical health or emotional problems make it difficult for you to do the following?" Response choices were "all of the time (100\%)," "most of the time," "half of the time $(50 \%), "$ "some of the time," and "none of the time $(0 \%) . "$ The response options "most of the time" and "some of the time" were recoded to $75 \%$ and $25 \%$ of the time, respectively, and "does not apply to my job" and blank responses were set to missing. Survey items were combined into 4 work limitation scales: time management (ability to handle time and scheduling demands of the job), physical work (ability to perform job tasks involving bodily strength, movement, endurance, coordination, and flexibility), mental-interpersonal (ability to perform cognitive and interpersonal job tasks), and output (ability to produce work output in a high-quality or timely manner). ${ }^{8}$ Categorical response options were converted to percentages and resulting scale scores ranged from 0 (limited none of the time in the past 2 weeks) to 100 (limited all of the time in the past 2 weeks).

To calculate percent productivity loss due to presenteeism, an index score was created by calculating a weighted sum from the 4 work limitation scales. The index score was then converted to a WLQ Loss Score, interpreted as the percentage of productivity loss in the past 2 weeks because of presenteeism relative to a healthy benchmark sample. ${ }^{8}$

Table 1. Definitions of Health Risk Categories

Risk Factor High-Risk Criteria

Obesity/overweight Smoker

Blood pressure

Cholesterol

Health Risk Categories

Low Risk

Medium Risk

High Risk
Reported height and weight equaling body mass index $\geq 27.5$

Described cigarette smoking habits as "still smoke"

Reported systolic blood pressure $>139 \mathrm{mmHg}$ or diastolic $>89$, or currently have high blood pressure, or currently take medication, or under medical care for blood pressure

Reported total cholesterol $>239 \mathrm{mg} / \mathrm{dL}$, or currently have high cholesterol, or currently take medication

Reported 0 to 2 health risks

Reported 3 to 4 health risks

Reported 5 or more health risks 


\section{Monetization of productivity loss}

Absenteeism costs were estimated using the "lost wages method," the most frequently used method to measure productivity loss. ${ }^{3,11}$ To derive the cost of work loss attributable to absenteeism in the past year, the total number of workdays lost was multiplied by the average daily compensation for full-time employees and an average wage "multiplier" of 1.61, where the multiplier is defined as the cost to an employer of an absence as a proportion of the absent worker's daily wage. ${ }^{12}$ Total daily compensation was based on average hourly wages plus benefits for civilian workers in the United States in 2008 (\$29.18 per hour for all US companies), multiplied by 8 hours per workday. ${ }^{13}$

Presenteeism costs were calculated by extrapolating estimates of productivity loss in the past 2 weeks (the WLQ Loss Score) to a 1-year time period in days (assuming 240 eligible work days/year), ${ }^{6}$ multiplied by total daily compensation.

To provide average-size employers with a benchmark for potential productivity loss, self-reported condition prevalence rates were multiplied by 10,000 (the average number of employees across OptumHealth "book of business" employer clients) to determine a typical number of employees reporting a condition or risk factor. Average annual productivity loss per year was calculated by multiplying the average number of people reporting a condition and the annual cost of productivity loss for each condition or risk, then summing across conditions. One year was used as a reference point for monetization because most employers evaluate health and productivity data over a 1-year time frame. ${ }^{6}$

\section{Estimates of employer medical costs}

To give employers a sense of the relative amount of productivity costs to medical costs, average annual allowed medical claims costs for each health condition and health risk measured by the HRA were estimated using Episode Treatment Groups (ETG) benchmark data. ${ }^{14}$ Allowed costs include the health plan liability (net paid amount) and patient liability (coinsurance and deductible amounts). ETG data are organized into clinically homogenous annualized episodes of care including inpatient, outpatient, ancillary, and pharmacy services. For chronic conditions, 1 episode typically corresponds to 1 individual. ETG data also include annual prevalence rates, episode-specific severity codes, and total cost for each treatment episode for several conditions. Annualized costs for a condition included costs for any comorbidities associated with an episode.

For each condition, the estimated average number of episodes was determined by multiplying the ETG benchmark prevalence rate by 10,000 employees. Medical costs were calculated by determining the weighted average cost per episode per condition, then multiplying cost by the average number of episodes. Pain and cigarette use were excluded from medical cost calculations because costs for chronic pain are subsumed by many ETGs, and because no ETG exists for medical costs related to smoking.

\section{Analyses}

Results from 2 different samples were analyzed. First, descriptive information-including prevalence of health conditions, comorbidities, health risks, and magnitude of absenteeism and presenteeism-was provided about all participants, even those who chose to respond to the HRA at only 1 point in time. In addition, further analyses were conducted for the cohort sample that completed 2 HRAs. The cohort sample was used to determine whether differences in health status and productivity loss were observed over time and to calculate productivity costs.

To determine productivity loss associated with specific health conditions and risks, propensity score analysis and a matching technique developed at the Mayo Clinic were employed. ${ }^{15}$ This method reduces bias and approximates a randomized trial by controlling for measurable differences between those with and those without health conditions and health risks, resulting in similar matched populations. ${ }^{16}$

First, the propensity score for each individual was created using a logistic regression model to estimate the probability that an individual would have a health condition or health risk based on his or her age, sex, season, and presence of other conditions and health risks (comorbidities). Individuals were then matched on these predicted probabilities using a greedy algorithm approach. Cases (participants with a particular health condition or health risk) were matched to controls (participants without the condition or risk) on the best match available. ${ }^{16}$ The resulting analysis groups differed only by presence of a particular health condition or risk. Individuals with conditions and controls who were not matched were eliminated from the sample.

Differences between cases and controls were assessed before and after matching using chi-square tests for categorical variables and $t$ tests for continuous variables to test for statistically significant differences between matched cases and controls. For analysis of productivity loss, those at low risk and who reported no health conditions were excluded from the majority of the models. Excluding healthy individuals from analysis may result in more accurate estimates of costs associated with health conditions because even when a condition is optimally managed, an individual will likely still exhibit productivity loss at rates different than someone without a condition. For analysis of allergies, high blood pressure, and overweight/obesity, however, healthy individuals were included in the models because not enough controls remained to match on propensity scores when they were removed.

Separate generalized linear models were used to estimate the productivity burden for each health condition and risk factor. This second-stage regression used total productivity expenditures at T2 as the dependent variable. Independent variables included any covariates that remained significantly different between the groups after matching, plus a dummy indicator to denote condition/risk status and total productivity expenditures at $\mathrm{T} 1$ to protect against chance imbalances in baseline productivity loss. Net lost productivity by condition/risk was calculated as the difference in lost productivity costs between the condition group (cases) and the matched control group. All analyses were performed using SAS software version 9.1.3 (SAS Institute Inc., Cary, $\mathrm{NC})$.

\section{Results}

Over half (56\%) of all participants reported having at least 1 health condition, and 31\% reported being at high risk for 
Table 2. Self-Reported vs. Benchmark Prevalence of Health Conditions and Health Risks

\begin{tabular}{|c|c|c|c|}
\hline \multirow[b]{2}{*}{ Condition/Risk } & \multicolumn{2}{|c|}{ Health Risk Appraisal } & \multirow{2}{*}{$\begin{array}{c}\text { Episode Treatment Groups Benchmark } \\
\% \text { with Condition }\end{array}$} \\
\hline & \% Reported Have Currently & \% Reported Have Comorbid Condition & \\
\hline Allergies & $28 \%$ & $58 \%$ & $5 \%$ \\
\hline Arthritis & $11 \%$ & $80 \%$ & $9 \%$ \\
\hline Asthma & $5 \%$ & $87 \%$ & $4 \%$ \\
\hline Back pain & $15 \%$ & $76 \%$ & $9 \%$ \\
\hline Bronchitis & $1 \%$ & $90 \%$ & $7 \%$ \\
\hline Cancer & $<1 \%$ & $71 \%$ & $2.5 \%$ \\
\hline Depression & $8 \%$ & $77 \%$ & $4 \%$ \\
\hline Diabetes & $5 \%$ & $64 \%$ & $4 \%$ \\
\hline Heartburn & $12 \%$ & $74 \%$ & $4 \%$ \\
\hline Heart disease & $2 \%$ & $78 \%$ & $5 \%$ \\
\hline Migraine & $7 \%$ & $73 \%$ & $2 \%$ \\
\hline Osteoporosis & $2 \%$ & $79 \%$ & $1 \%$ \\
\hline Pain & $6 \%$ & $93 \%$ & NA \\
\hline Overweight/Obese & $44 \%$ & $54 \%$ & $1 \%$ \\
\hline Smoker & $10 \%$ & $55 \%$ & NA \\
\hline High blood pressure & $20 \%$ & $58 \%$ & $12 \%$ \\
\hline High cholesterol & $17 \%$ & $59 \%$ & $12 \%$ \\
\hline
\end{tabular}

health problems. On average, participants reported 1 health condition (range: 0 to 13) and 1.96 risks (range: 0 to 13). Age, sex, and several comorbid conditions were significantly associated with having health conditions and health risks. Allergies were the most prevalent health condition and overweight/obesity was the most prevalent risk factor (Table 2). Prevalence estimates for the selected conditions were fairly similar when comparing ETG data with self-reported HRA data, with less than a 10 percentage point difference between highest and lowest prevalence estimates for most conditions and risks. However, prevalence estimates for allergies ranged from a low of 5\% for ETG data to a high of $28 \%$ reported by HRA participants, and for obesity, estimates ranged from $1.4 \%$ to $42 \%$ for ETG vs. HRA data, respectively (Table 2 ).

Across all health conditions and risk categories, participants reported an annual average of 1.99 days of work missed due to illness and 9.04 days at work but limited in performing tasks because of health. Presenteeism and absenteeism were significantly associated with presence of all health conditions and lifestyle health risks. Higher numbers of health risks and health conditions were associated with lower levels of productivity (Table 3 ).

A significant amount of change was seen in the number of self-reported conditions from T1 to T2 (Table 4). In addition, increases and decreases in the number of health conditions were associated with same direction changes in productivity; that is, those who reported having fewer conditions at T2 also reported a reduction in productivity loss and those who reported an increase in the number of conditions from $\mathrm{T} 1$ to $\mathrm{T} 2$ also reported an increase in productivity loss.

Average calculated costs of productivity loss (including both absenteeism and presenteeism) were higher for those with conditions and those at high risk for health problems compared with matched controls; cancer, bronchitis, and depression were among the top 3 conditions in terms of productivity costs (Fig. 1).

Combining productivity costs and ETG medical costs across conditions and risks reported on the HRA (except pain and smoking) for an average-size employer showed that for every dollar of medical costs there were 0.4 dollars of productivity costs (Fig. 2).

\section{Discussion}

This article presents the results from a comprehensive evaluation of OptumHealth HRA data in an effort to determine the relationship between health status and productivity loss among employed participants. After matching and regression-based adjustments were made, employees with health conditions and at high risk for health problems had

Table 3. Annual Productivity loss by Number of Health Conditions and Risk Level

\begin{tabular}{|c|c|c|c|c|}
\hline & Number & $\%$ & Mean Annual Absent Days & Mean Annual Unproductive Days \\
\hline \multicolumn{5}{|l|}{ Condition Level } \\
\hline No conditions & 563,506 & $45 \%$ & 1.4 & 3.7 \\
\hline 1 condition & 361,843 & $29 \%$ & 1.9 & 7.9 \\
\hline $2+$ conditions & 338,768 & $27 \%$ & 3.0 & 20.1 \\
\hline \multicolumn{5}{|l|}{ Risk Level } \\
\hline Low (0-2 risks) & 870,457 & $68 \%$ & 1.6 & 5.1 \\
\hline Medium (3-4 risks) & 271,404 & $21 \%$ & 2.4 & 12.9 \\
\hline High $(5+$ risks $)$ & 129,028 & $10 \%$ & 3.6 & 28.9 \\
\hline
\end{tabular}


Table 4. Overall Change in Prevalence of Self-Reported Health Conditions: Time 1 to Time 2

\begin{tabular}{cccc}
\hline Stayed the Same (no health condition) & Got Worse & Got Better & Stayed the Same (with health condition) \\
\hline $35 \%$ & $9 \%$ & $10 \%$ & $45 \%$ \\
\hline
\end{tabular}

productivity costs ranging from $\$ 15$ to $\$ 1601$ more per year than similar employees without health conditions/risks. This means that an employer with 10,000 employees could face nearly $\$ 3.8$ million in productivity loss each year, in addition to medical costs for these conditions.

The condition-specific productivity cost estimates noted here are similar to estimates found in previous studies for some conditions, but are higher or lower for other conditions. ${ }^{1,6}$ Differences in productivity costs may be attributable to different instruments used to measure work loss, differing study designs (eg, others did not match those with conditions to a comparison group based on propensity scores), and/or differing monetization methods (eg, healthy individuals were excluded from productivity cost estimates, the wage multiplier was assumed to be inappropriate for presenteeism).

In addition to potential differences in study designs and monetization methods, the average medical to productivity cost ratio may differ from results reported in other studies for a variety of possible reasons, including matching actual medical claims to the cohort of HRA respondents was beyond the scope of this project and doing so may have resulted in a different proportion of productivity to medical expenditures; pain (a high-cost health condition) was excluded from productivity and medical cost comparisons; comorbidities were controlled for in productivity cost analysis but were included in benchmark medical costs; the prevalence of health conditions in the population analyzed in this study may differ from prevalence rates of conditions in populations evaluated in other studies; or the variability in prevalence of conditions across data sources (HRA vs. ETG) may have resulted in lower than actual medical costs. Given the impracticality of evaluating individual medical claims and HRA data for all business purchasers of health management services, however, these benchmark results can help employers estimate their total health-related costs and highlight the importance of preventing and managing diseases and health risks effectively.

Overall, the current findings are consistent with other studies that have found that health risks and health condi-

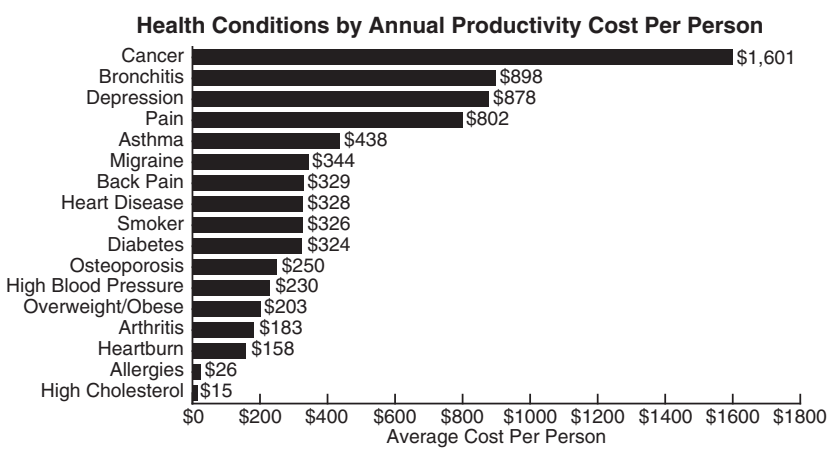

FIG. 1. Health conditions by annual productivity cost per person. tions are related to productivity losses. ${ }^{1,3,6,10}$ Findings also underscore the need for standardized measures for productivity loss and methods for monetizing that loss so that costs can be compared across studies.

These analyses have several limitations. Given that the distribution of job categories among participants was unknown, a multiplier of 1.61 was used to monetize absenteeism based on the mean absence multiplier reported in the Nicholson study. ${ }^{15}$ Actual costs of absences may differ by industry and employers. In addition, propensity score matching is never perfect; it was not possible to include in the models all the variables related to having a health condition and related outcomes or to control for non-healthrelated variables that are sources of workplace productivity loss. Because the intent was to estimate productivity loss on an annual basis, presenteeism estimates were converted to annual rates. Extrapolating values from shorter time periods to values that extend to 1 year could underestimate or overestimate time losses. ${ }^{6}$ Finally, the monetization of productivity loss itself necessitates caution resulting from lack of consensus in the health management community about monetization methodology for presenteeism. ${ }^{2}$

Despite these limitations, this study applied a robust evaluation design with a large sample of subjects to determine the implications of lost work performance. Further research is needed to determine the effects of treatment on productivity loss and whether savings could be realized by optimally managing the health risks and conditions included in this analysis.

\section{Conclusion}

Results of this study support the premise that health conditions and lifestyle risk factors are associated with workplace productivity loss and reiterate the value of maintaining a healthy population. We determined practical estimates of the costs of productivity loss related to health.

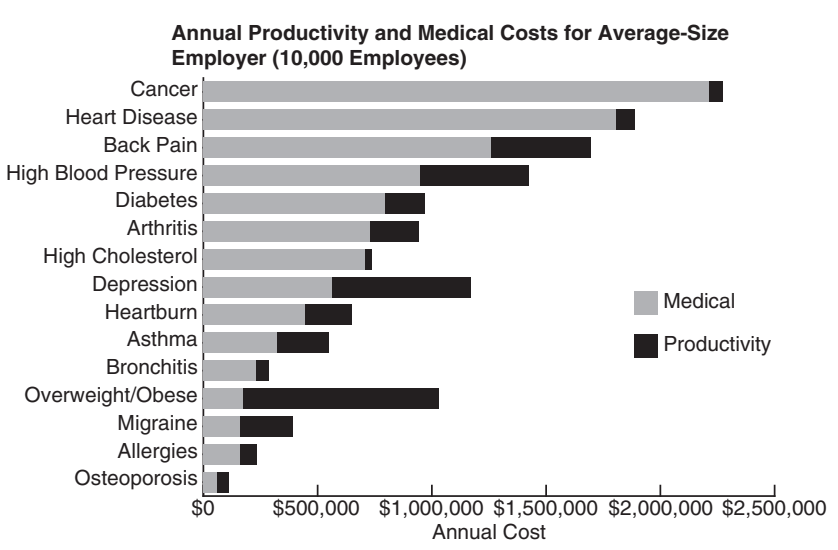

FIG. 2. Annual productivity and medical costs for averagesize employer (10,000 employees). 
This view of productivity loss across several conditions and health risks may help employers assess the impact of productivity loss in their own companies.

In the absence of standardized methods for monetizing productivity loss, the cost estimates of lost work time presented here suggest areas where employers could concentrate efforts to address health risks and chronic conditions to improve productivity-related financial outcomes.

\section{Author Disclosure Statement}

Ms. Mitchell and Dr. Bates disclosed no conflicts of interest.

\section{References}

1. Loeppke R, Taitel M, Haufle V, Parry T, Kessler RC, Jinnett $\mathrm{K}$. Health and productivity as a business strategy: A multiemployer study. J Occup Environ Med 2009;51:411-428.

2. Disease Management Association of America. Outcomes Guidelines Report, Volume 4. Washington, DC: DMAA: The Care Continuum Alliance, Inc.; 2009.

3. Mattke S, Balakrishnan A, Bergamo G, Newberry SJ. A review of methods to measure health-related productivity loss. Am J Managed Care 2007;13:211-217.

4. Davis K, Collins SR, Doty MM, Ho A, Holmgren A. Health and productivity among U.S. workers. Available at: http:// www.commonwealthfund.org/ /media/Files/Publications/ Issue $\% 20 B r i e f / 2005 / \mathrm{Aug} / \mathrm{Health} \% 20$ and $\% 20$ Productivity $\%$ 20Among\%20U\%20S\%20\%20Workers / 856_Davis_hlt_ productivity_USworkers\%20pdf.pdf. Accessed May 23, 2010.

5. Towers Watson. 2009/2010 North American Staying@Work Report: The Health and Productivity Advantage. New York: Towers Watson; 2009.

6. Goetzel RZ, Long SR, Ozminkowski RJ, Hawkins K, Wang S, Lynch W. Health, absence, disability, and presenteeism cost estimates of certain physical and mental health conditions affecting U.S. employers. J Occup Environ Med 2004; 46:398-412.

7. Short ME, Goetzel RZ, Pei X, et al. How accurate are selfreports? Analysis of self-reported health care utilization and absence when compared with administrative data. J Occup Environ Med 2009;51:786-796.

8. Lerner D, Rogers WH, Chang H. Scoring the Work Limitations Questionnaire (WLQ) Scales and the WLQ Index for Estimating Work Productivity Loss. Boston, MA: The Health Institute of the Tufts-New England Medical Center, 2005.

9. Prasad M, Wahlqvist P, Shikiar R, Ya-Chen TS. A review of self-report instruments measuring health-related work productivity. Pharmacoeconomics 2004;22:225-244.

10. Schultz AB, Edington DW. Employee health and presenteeism: A systematic review. J Occup Rehabil 2007;17: 547-579.

11. Berger ML, Murray JF, Xu J, Pauly M. Alternative valuations of work loss and productivity. J Occup Environ Med 2001; 43(1):18-24.

12. Nicholson S, Pauly MV, Polsky D, Sharda C, Szrek H, Berger ML. Measuring the effects of work loss on productivity with team production. Health Econ 2006;15:111-123.

13. United States Department of Labor. Employer costs for Employee Compensation: December 2008. Washington, DC: U.S. Bureau of Labor Statistics; 2008.

14. Ingenix, Inc. Symmetry Episode Treatment Groups: Measuring Health Care with Meaningful Episodes of Care. Eden Prairie, MN: Ingenix; 2008.

15. Bergstralh EJ, Kosanke JL. Computerized Matching of Cases to Controls. Technical Report Number 56. Rochester, MN: Mayo Foundation; 1995.

16. Parsons LS. Reducing bias in a propensity score matchedpair sample using greedy matching techniques. Poster presented at: SUGI 26 Proceedings; Long Beach, CA; April 22-25, 2001. 